\title{
Radiologic Evaluation of Degeneration in Isthmic and Degenerative Spondylolisthesis
}

\author{
Hyun-Yoon Jeong, Jae-Won You, Hong-Moon Sohn, Sang-Ha Park \\ Department of Orthopaedic Surgery, Chosun University College of Medicine, Gwangju, Korea
}

\begin{abstract}
Study Design: A cross-sectional imaging study.
Purpose: The objective was to assess the degree of degeneration and the associated factors through imaging studies of the lesion segment and the adjacent superior and inferior segments of isthmic and degenerative spondylolisthesis.

Overview of Literature: Few articles existed for degeneration and related factors in isthmic and degenerative spondylolisthesis.

Methods: The subjects were 95 patients diagnosed with spondylolisthesis. Simple plain radiographs including flexion and extension and magnetic resonance imaging were used to investigate the degree of translation, disc degeneration, high intensity zone (HIZ) lesion, Schmorl's node (SN) and Modic changes.

Results: Advanced disc degeneration, grade 5 , was shown to be significant in the index segment of the isthmic type $(p=0.034)$. Overall, type 2 Modic change was most common in both groups and also, it was observed more in the isthmus group, specifically, the index segment compared to the degenerative group $(p=0.03)$. For the $S N$, compared to the degenerative type, the isthmus type had a significantly high occurrence in the index segment $(p=0.04)$. For the HIZ lesions, the isthmus type had a higher occurrence than the degenerative type, especially in the upper segment $(p=0.03)$.

Conclusions: Most advanced disc degeneration, fifth degree, SN and Modic change occurred more frequently in the lesions of the isthmus type. HIZ lesions were observed more in the isthmus type, especially in the segment superior to the lesion.
\end{abstract}

Keywords: Lumbar vertebrae; Spondylolisthesis; Degeneration; Radiography

\section{Introduction}

Spondylolisthesis is defined as a condition where there is a translational displacement in the upper vertebral body compared to the lower vertebral body. Since the year 1782, after the gynecologist Herbiniaux [1] first described it, there have been many efforts to classify the condition based on clinical symptoms and anatomical form.

In 1976, Wiltse et al. [2] classified five types of con- genital, isthmic, degenerative, traumatic, and pathologic spondylolisthesis. In 1989, Wiltse and Rothman [3] separated the post-surgical type from the pathologic type producing 6 different classifications for spondylolisthesis, which is the common form used today.

The central and lateral recess type of spinal stenosis which was led by facet hypertrophy, protrusion of disc, and hypertrophy of the ligament flavum had been commonly shown in spondylolisthesis. Also, this is related to

Received Oct 4, 2012; Revised Dec 14, 2012; Accepted Jan 10, 2013

Corresponding author: Jae-Won You

Department of Orthopaedic Surgery, Chosun University College of Medicine,

365 Pilmun-daero, Dong-gu, Gwangju 501-717, Korea

Tel:+ 82-62-220-3147, Fax: +82-62-226-3379, E-mail: jwyou@chosun.ac.kr 
degeneration closely. The isthmic type and the degenerative type are the most frequent types, but there were few articles for degeneration and related factors in adjacent segments and lesion segments of isthmic and degenerative spondylolisthesis.

We assessed the degree of degeneration and the associated factors through simple plain radiographs and magnetic resonance imaging (MRI) of the lesion segments and the adjacent superior and inferior segments of isthmic and degenerative spondylolisthesis.

\section{Materials and Methods}

\section{Subjects}

The subjects included 95 people who have taken simple plain radiographs including flexed and extended views and MRI out of the patients that have been recently diagnosed with spondylolisthesis. They xhibited symptoms such as low back pain, claudication or radiating pain while visiting our clinic. There were 46 cases of the isthmus type and 49 cases of the degenerative type and the average age was 53.6 years (range, 19 to 77 years) and 63.5 years (range, 32 to 82 years) each, respectively. For the isthmus type there were 16 men and 30 women and there were 29 cases (63\%) for L5-S1, 15 cases for L4-5 and 2 cases for L3-4. For the degenerative type there were 15 men and 34 women and there were 34 cases (69.4\%) for L4-L5, 3 cases for L3-4 and 12 cases for L5-S1. Patients that have had lumbar surgery or a vertebral fracture and patients that show spondylolisthesis in multiple segments or manifest both types of the condition have been excluded from the research.

\section{Plain radiographs}

Degrees of translational displacement were classified into stages 1 through 5, using the Meyerding [4] classification (Fig. 1A). Angular and translational segment motions were acquired in flexion-extension radiological images. The degree of translation between segments was defined as the relative forward protrusion of the upper vertebral body comparison to the lower vertebral body and was recorded in $\mathrm{mm}$. The difference in segment angles was defined as the difference in the angle of the line drawn along the upper margin of each vertebral body (Fig. 2). A translation greater than $3 \mathrm{~mm}$ and angular motion beyond $10^{\circ}$ were defined as being unstable, which required surgical treatment [5].

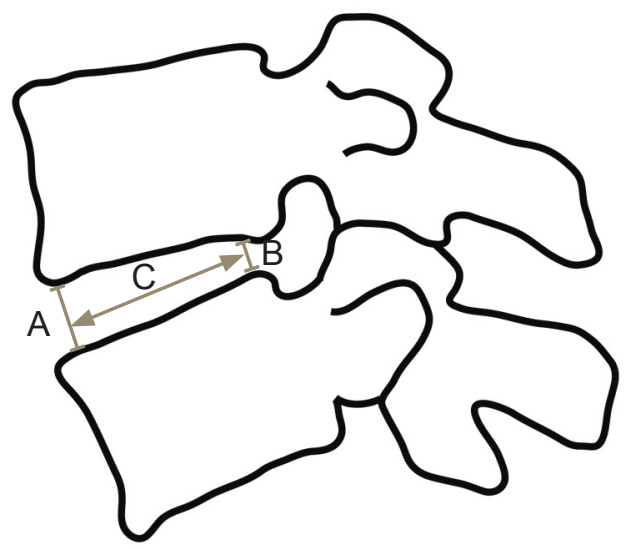

$$
\begin{aligned}
& \text { Disc space height } \\
& =(A+B) / C
\end{aligned}
$$

Fig. 1. (A) Measurement of slip by Meyerding scale. (B) measurement of disc height as Farfan index. The sum of anterior disc height $A$ and posterior height $B$ is divided by sagittal disc width $C$. 


\section{Magnetic resonance imaging}

Using a Siemens Avanto 1.5T MRI (Siemens, Munchen, Germany) sagittal T1-weighted images (758/12 [repetition time/echo time msec]) and sagittal T2-weighted images $(4,667 / 112)$ were acquired. Each had a thickness of 4 $\mathrm{mm}$ and the matrix for $\mathrm{T} 1$ and $\mathrm{T} 2$ weighted images were $168 \times 512,180 \times 512$, respectively.

\section{Degeneration of the disks}

Using the modified Pfirrmann classification [6], the degree of degeneration of the disks shown in the sagittal T2-weighted image were classified as stage 1 (normal shape, no horizontal band, distinctive nucleus pulposus and normal annulus), stage 2 (heterogeneous shape of nucleus pulposus with a horizontal band and vague distinction between the nucleus pulposus and the annulus), stage 3 (vague distinction between the heterogeneous nucleus pulposus and the annulus, but still identifiable), stage 4 (heterogeneous nucleus pulposus with a low signal intensity, annulus rupture and typically, decrease in disk height) and stage 5 (same as stage 4 with collapse of intervertebral space). Disk degeneration was defined as stage 3 or worse. Also, advanced disc degeneration was defined by grade 5 .

\section{Modic change}

Using the method recommended by Modic et al. [7], Modic change, depending on the existence of change in the vertebral body end plate and bone-marrow, was classified as type I (showing low signal intensity in a T1weighted image and high signal intensity in a T-2 weighted image), type II (high signal intensities in both T1 and T2-weighted images) and type III (low signal intensities in both $\mathrm{T} 1$ and $\mathrm{T} 2$-weighted images).

\section{Schmorl's node, high intensity zone lesion, disc height}

Schmorl's nodes (SN) are defined as localized irregular shapes of the endplates superior and inferior to the vertebral disk and disk herniations into the vertebral body. HIZ lesions are defined as localized high signal intensities in the region posterior to the annulus fibrosus in T2weighed images. The heights of the disks were obtained using the Farfan index [8] to minimize the error by measuring the disk heights from each anterior and posterior regions in sagittal T2-weighted images and dividing the addition of the two measurements by the diameters of the disks (Fig. 1B).
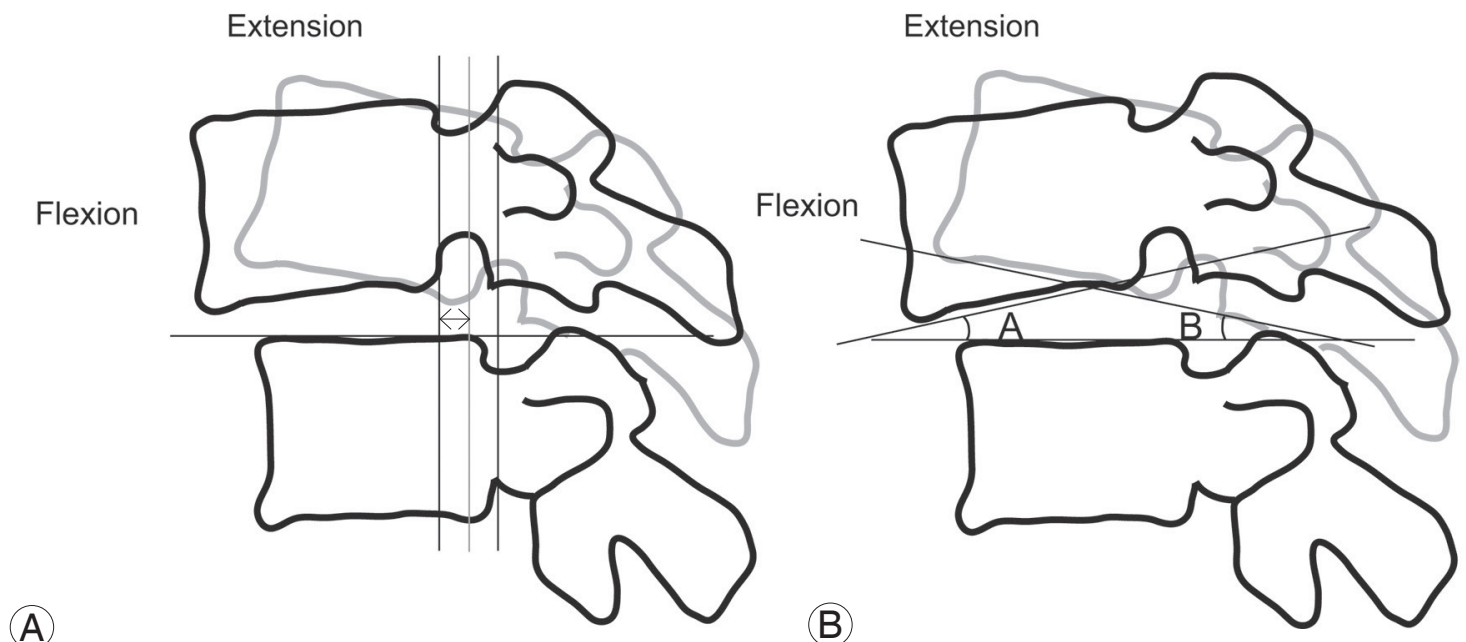

Fig. 2. Measurement of segmental motion. (A) Segmental translation. A base line is drawn at the superior endplate of lower vertebra. Also, the perpendicular line to the base line at the posteroinferior corner of the upper vertebra is drawn on the flexion and extension radiograph. The difference (arrow) is calculated. (B) Segmental angulation. The angle between the line on the upper endplate of lower vertebra and the line on the lower endplate of upper vertebra is measured on flexion and extension radiographs. In addition, the difference $(\mathrm{B}-\mathrm{A})$ is calculated. 
Table 1. Patient demography

\begin{tabular}{|c|c|c|}
\hline & $\begin{array}{l}\text { Isthmic type } \\
\text { (46 case) }\end{array}$ & $\begin{array}{c}\text { Degenerative type } \\
\text { (49 case) }\end{array}$ \\
\hline Age (yr) & 53.6 (19-77) & 63.5 (32-82) \\
\hline \multicolumn{3}{|l|}{ Sex } \\
\hline Male & 16 & 15 \\
\hline Female & 30 & 34 \\
\hline \multicolumn{3}{|l|}{ Level } \\
\hline L3-4 & 2 & 3 \\
\hline L4-5 & 15 & 34 \\
\hline L5-S1 & 29 & 12 \\
\hline \multicolumn{3}{|c|}{ Meyerding grade } \\
\hline Grade 1 & 30 & 42 \\
\hline Grade 2 & 15 & 6 \\
\hline Grade 3 & 1 & 0 \\
\hline Grade 4 & 0 & 1 \\
\hline
\end{tabular}

\section{Data analysis}

Each parameter was measured twice by one orthopedic surgeon and intraobserber reliability was determined using kappa values. We used IBM SPSS ver. 20 (IBM Corp., Armonk, NY, USA). In addition, we use an independent t-test and Pearson chi-square test for data analysis. $p$ values below 0.05 indicated statistical significance.

\section{Results}

There were 46 cases for the isthmus type, 16 cases being men. The average age was 53.6 years (range, 19 to 77 years) and there were 29 cases (63\%) for L5-S1, 15 cases for L4-5 and 2 cases for L3-4. For the degenerative type there were 49 cases, out of which 34 were women. There were 34 cases (69.4\%) for L4-L5, 3 cases for L3-4 and 12 cases for L5-S1, L4-L5 being the most common region (Table 1). Furthermore, intraobserver agreement was almost perfect at $92 \%(\mathrm{k}: 0.83)$.

\section{Degrees of translation}

The Meyerding method was used to measure the translational displacement and in the isthmus type there were 30 cases of grade 1, 15 cases for grade 2 and 1 case for grade 3. Grade 4 and 5 were not observed, as most cases were grade 1 or 2 . In the degenerative type there were 42 cases of grade 1, 6 cases of grade 2 and 1 case of grade 4 . Grade 3 and 5 were not observed and most were grade 1 or 2 (Table 1).

\section{Translational and anglular and instability}

For the upper segment (46 cases) in the isthmus type, the average horizontal translation was $2.0 \mathrm{~mm}$ and there were 12 unstable cases. The average angular movement was $7.7^{\circ}$ and 13 cases were unstable. For the index segment (46 cases), the average horizontal translation was $1.5 \mathrm{~mm}$, there were 8 unstable cases, the average angular movement was $7.8^{\circ}$ and there were 15 unstable cases. For the lower segment (46 cases) the average horizontal translation was $0.7 \mathrm{~mm}$, there was no unstable case, the average angular movement was $7.9^{\circ}$ and there were 6 unstable cases. There was no statistical significance between the two types of spondylolisthesis $(p>0.05)$ (Table 2).

\section{Disk degeneration}

Comparing the isthmus type and the degenerative type, the number of cases defined as disk degeneration was 41 cases $(89.1 \%)$ and 45 cases $(91.8 \%)$ for the upper segment, $44(95.7 \%)$ cases and 41 cases $(83.7 \%)$ for the index segment and 11 cases (57.9\%) and 32 cases (80.0\%) for the lower segment, respectively. There was no statistical significance between the two types of spondylolisthesis $(p>0.05)$. However, the most advanced disc degeneration, fifth grade, were shown to be significant in the index segment of the isthmic type ( $p=0.034)$ (Table 3$)$.

\section{Disk height}

The Farfan index 7 was used in midsagittal T2-weighted MRI images in order to minimize the error when measuring the disk heights. The values obtained for the isthmus type and the degenerative type when using this method were 0.44 and 0.38 for the upper segment, 0.39 and 0.38 for the index segment and 0.42 and 0.46 for the lower segment, respectively. There was no statistical significance between the two types of spondylolisthesis ( $p=0.07, p=0.83, p=0.52$ ). The relationship between disk degeneration and disk height was significant only in the upper segment $(p=0.01)$. 
Table 2. Segmental translation motion and segmental angular motion

\begin{tabular}{|c|c|c|c|}
\hline & Isthmic type & Degenerative type & $p$-value \\
\hline \multicolumn{4}{|c|}{ Translation motion, mm (case) } \\
\hline Upper (instability) ${ }^{\text {a) }}$ & $2.0(12)$ & $1.7(6)$ & 0.085 \\
\hline Index (instability) & $1.5(8)$ & $1.2(6)$ & 0.479 \\
\hline Lower (instability) & $0.7(0)$ & $1.2(6)$ & 0.078 \\
\hline \multicolumn{4}{|c|}{ Angular motion, ${ }^{\circ}$ (case) } \\
\hline Upper (instability) & $7.7(13)$ & $6.5(8)$ & 0.161 \\
\hline Index (instability) & $7.8(15)$ & $7.2(11)$ & 0.267 \\
\hline Lower (instability) & $7.9(6)$ & $7.0(11)$ & 0.638 \\
\hline
\end{tabular}

a) Instability criteria: translation $>3 \mathrm{~mm}$, or segmental angular difference $>10^{\circ}$.

Table 3. Disc degeneration in isthmic and degenerative spondylolisthesis

\begin{tabular}{lccc} 
& Isthmic type & Degenerative type & $p$-value \\
Degenerationa) & & & \\
\hline Upper segment & $41(89.1)$ & $45(91.8)$ & 0.070 \\
\hline Index segment & $44(95.7)$ & $41(83.7)$ & 0.090 \\
\hline Lower segment & $11(57.9)$ & $32(80)$ & 0.110 \\
\hline Advanced degeneration (grade 5) & & $3(7.5)$ & 0.613 \\
\hline Upper segment & $1(5.3)$ & $7(14.3)$ & 0.034 \\
\hline Index segment & $15(32.6)$ & $3(6.1)$ & 0.149 \\
\hline
\end{tabular}

Values are presented as number (\%).

a)Degeneration means Pfirmann grade $\geq 3$.

Table 4. Modic change in isthmic and degenerative spondylolisthesis

\begin{tabular}{|c|c|c|c|c|c|}
\hline \multirow{2}{*}{ Modic change } & \multicolumn{2}{|c|}{ Isthmic type } & \multicolumn{2}{|c|}{ Degenerative type } & \multirow{2}{*}{$p$-value } \\
\hline & $A l^{a)}$ & Subtype (I:II:III) & All & Subtype (I:II:III) & \\
\hline Upper & $4(8.7)$ & 2:1:1 & $7(14.3)$ & $1: 4: 2$ & 0.395 \\
\hline Index & $24(52.2)$ & 7:15:2 & $11(22.4)$ & 1:8:2 & 0.003 \\
\hline Lower & $5(29.4)$ & 0:5:0 & $7(18.9)$ & 2:4:1 & 0.389 \\
\hline
\end{tabular}

Values are presented as number (\%).

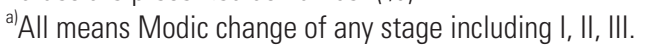

\section{Modic change}

For the isthmus type, Modic change was observed in 4 cases $(8.7 \%)$ in the upper segment, 24 cases in the index segment $(52.2 \%)$ and 5 cases in the lower segment (29.4\%). For the degenerative type, there were 7 cases $(14.3 \%)$ in the upper segment, 11 cases $(22.4 \%)$ in the index segment and 7 cases (18.9\%) in the lower segment. For the subtypes of Modic change of the isthmus type, there were 9 cases for type 1, 21 cases for type 2 and 3 cases for type 3 and for the degenerative type there were 4 cases for type 1, 16 cases for type 2 and 5 cases for type 3 . Overall, Modic change was most common in both groups and also, it was observed more in the isthmus group, specifically, in the index segment compared to the degenerative group ( $p=0.03$ ) (Table 4 ). 
Table 5. Schmol's node in isthmic and degenerative spondylolisthesis

\begin{tabular}{lccc} 
& Isthmic type & Degenerative type & $p$-value \\
Schmorl's node & & & \\
Upper segment & $6(13)$ & $20(18.4)$ & 0.477 \\
\hline Index segment & $28(60.9)$ & $6(39.5)$ & 0.041 \\
Lower segment & $6(35.6)$ & $33(67.3)$ & 0.768 \\
\hline Present in any segment ${ }^{\text {a) }}$ & $32(69.6)$ & 0.816 \\
\hline
\end{tabular}

Values are presented as number (\%).

alPresent in any segment means that is a general prevalence of $\mathrm{SN}$ in all segments including upper, index and lower segment.

Table 6. HIZ lesion in isthmic and degenerative spondylolisthesis

\begin{tabular}{lccc} 
& Isthmic type & Degenerative type & $p$-value \\
HIZ lesion & & & $6(12.2)$ \\
Upper segment & $18(39.1)$ & $23(46.9)$ & 0.003 \\
Index segment & $28(60.9)$ & $8(21.6)$ & 0.174 \\
Lower segment & $5(29.4)$ & $30(61.2)$ & 0.733 \\
\hline Present in any segment & $38(82.6)$ & 0.021 \\
\hline
\end{tabular}

Values are presented as number (\%).

$\mathrm{HIZ}$, high intensity zone.

\section{Schmorl's node}

When the $\mathrm{SN}$ were observed in more than one segment, there were 32 cases (69.9\%) in the isthmus type and 33 cases $(67.3 \%)$ in the degenerative type and there was no statistical significance between the two groups ( $p=0.816$ ). However, when comparing the isthmus type and the degenerative type at each segment, there were 6 cases $(13.0 \%)$ and 9 cases (18.4\%) in the upper segment, 28 cases (60.9\%) and 20 cases (40.8\%) for the index segment and 6 cases (35.6\%) and 15 cases (39.5\%) in the lower segment, respectively. Compared to the degenerative type, the isthmus type had a significantly high occurrence in the index segment, where the lesion is located $(p=0.041)$ and there were no differences between the upper and lower segment ( $p>0.05)$. Also, within the isthmus group, there was statistical significance for high occurrence in the lesion segment (Table 5).

\section{High intensity zone lesion}

When HIZ lesions were observed in more than one segment, there were 38 cases $(82.6 \%)$ for the isthmus type and 30 cases $(61.23 \%)$ for the degenerative type and there was statistical significance for the difference between the two types of spondylolisthesis $(p=0.021)$. When com- paring the isthmus type and the degenerative type at each segment, there were 18 cases $(39.1 \%)$ and 6 cases (12.2\%) in the upper segment, 28 cases (60.9\%) and 23 cases (46.9\%) in the index segment, and 5 cases (29.4\%) and 8 cases $(21.6 \%)$ in the lower segment, respectively. Compared to the degenerative type, the isthmus type had a significantly high occurrence in the upper segment $(p=0.03)$ and there was no difference in the index and lower segment $(p>0.05)$ (Table 6$)$.

\section{Discussion}

In 1989, Wiltse and Rothman [3] have separated the post-surgical type from the pathologic type producing 6 different classifications for spondylolisthesis, which is the common form used today. The isthmic type and the degenerative type are the most frequent types and they differ in cause, developmental mechanism, natural development and treatment. The isthmus type is defined as a condition where there is fibrous loss in the isthmus of the posterior arch resulting in forward protrusion of the upper vertebral body and the anterior surface of the vertebral body being separated from the neural arch. Its main mechanism is thought to be repetitive with periodic anterior and posterior bending, especially, stress fracture due to extension [9]. For this type, there is a high inci- 
dence rate in men and it occurs frequently between the L5 and S1. In the case of adults, there is instability in the lesion, resulting in degenerative change, in turn causing nerve compression leading to severe pain and neurological symptoms, therefore, requiring surgical treatment. On the other hand, it was discovered by Newman and Stone [10] that the degenerative type is caused by degenerative change and instability of the lumbar causing hypertrophy of the bones and soft tissue, and as a result, causes back pain and neurological symptoms $[11,12]$. For this type, there is a high incidence rate in women and it occurs frequently between the L4 and L5. In most cases, symptoms can be alleviated through conservative treatment; however, if the condition is unresponsive to conservative treatment and pain persists surgical treatment is required [12]. The isthmic and the degenerative type of spondylolisthesis have many different features in terms of causes, pathogenesis, and natural history. However both types show progressive degeneration according to age. There are few studies concerning the degenerative features of the two types of spondylolisthesis. In this study, we assessed the degree of degeneration and the associated factors by analyzing simple plain radiographs and MRI of the lesion segment, adjacent superior and inferior segments of isthmic and degenerative spondylolisthesis.

In this study, there were 46 cases for the isthmus type, 16 of which were men and there were 29 cases (63\%) for L5-S1, being the most frequent segment of exposure. For the degenerative type there were 46 cases, out of which 34 were women and there were 34 cases (69.4\%) for L4L5. The reason for the abundance of women in both types is thought to be because there were more women (69.4\%) involved in the research.

The authors used the Meyerding method to measure the translational displacement. In the isthmus type and the degenerative type, altogether, there were 71 cases of stage 1, 22 cases for stage 2, as most cases were in stages 1 or 2. Previous studies have reported an increase of segmental instability in moderate to severe disc and posterior facet degeneration which improve when degeneration progresses [13]. Fujiwara et al. [14] reported segmental movement to be increased up to the fourth degree of disc degeneration and improve in the advanced fifth degree.

The horizontal translation and segment angle were measured in flexion and extension radiograph images. Segment horizontal translation was defined as the difference of translation in flexion and extension views and was recorded in units of mm. Angular segment motion was defined as the difference in the angle of the line drawn along the upper margin of each vertebral body in motion views. Cases where there were horizontal transpositions greater than $3 \mathrm{~mm}$ and angular movements greater than $10^{\circ}$ were assumed to be unstable and eventually needed surgical treatment. In this study, the numbers of cases defined as more advanced degeneration, fifth degree, was shown frequently in the isthmus type. This may propose that restabilization is progressed in the isthmic type, but the actual measured horizontal translation and segmental motion represented that there was no statistical significance between the two types of spondylolisthesis. This indicates that there may be other factors contributing to the restabilization of the segment.

There were 47 cases where the patient underwent operation and among them there were 33 cases $(70.2 \%)$ where the patients had instability. The rest were cases which the patients did not respond to conservative treatment. For the isthmus type and the degenerative type there were 23 cases $(48.9 \%)$ and 24 cases $(51.1 \%)$, respectively.

The classification by Pfirrmann was used on Disk degeneration while stages 3 or more was defined as degenerated. There was insufficient research done using MRI on disk degeneration within the lesion segment and the segments adjacent to the lesion of spondylolisthesis patients. Overall, for the disk degeneration, there was no difference in degenerative change between the two groups. Disc degeneration in the isthmus type had a significantly high occurrence in the index segment, where the lesion is located, and relatively low occurrence in the lower segment. This result is consistent in that advanced disc degeneration, fifth degree, was also most commonly observed in the index segment ( $p=0.034)$. For the degenerative type, disc degeneration was more common in the upper segment, but overall degeneration was similar in all segments. These differences did not have demonstrate statistical significance $(p>0.05)$.

As the vertebral disks age, they undergo degenerative change. The loss of fluid and elasticity and the decrease in height leads to instability of the vertebra which eventually causes translation of the vertebral body [15-17]. Disc degeneration occurs prior to posterior facet joint degeneration, and this loss of disc height and change of mechanical properties can lead to posterior facet joint degeneration and low back pain through increasing load to the facet joints [18-20]. 
Modic et al. [7] classified spondylolisthesis into type I (low intensity in T1-weighted images, high intensity in T-2 weighted images), type II (high intensity in both T1 and T2-weighted images) and type III (low intensity in both $\mathrm{T} 1$ and $\mathrm{T} 2$-weighted images) depending on the existence of change in the endplates and the bone marrow. Type I indicates active and ongoing degenerative process and type II represents a more stable and chronic degenerative form than type I. Type III is thought to be associated with subchondral bone sclerosis. Another research has associated type I and occurrences in the L5-S1 region with patients' symptoms [21]. In this research, type II was the most abundant form in both the isthmus type and the degenerative type. There were more changes in the isthmus type than the degenerative type, specifically, in the index segment where the lesion is located. As advanced disc degeneration is more commonly observed in the index segment, we can conclude that the lesion site for the isthmic type is more stable than the upper and lower segment of the lesion.

A HIZ lesion is thought to be radial and concentric tears of the posterior annulus [22]. It occasionally appears as mucoid material associated with fluid or granulation tissue, outer annulus with ruptured neovasculature or substances formed under a PLL (posterior longitudinal ligament) complex [23]. There is much controversy in the clinical significance of HIZ lesions. Aprill and Bogduk [22] have stated there is a significant relationship between the existence of HIZ and annular tear, however, Ricketson et al. [24] have mentioned there is no association between $\mathrm{HIZ}$ and concordant pain.

$\mathrm{SN}$ is defined as a localized irregular shape in the vertebral endplates encasing the disk or disk herniation into the vertebral body. Despite that the function of SN is not well known, it is thought that it plays a role in the degeneration of the disk [25-28]. Also, SN is more prevalent in males because of the higher physical loading [29], and this is associated with its higher occurrence in males with the isthmus type.

This research has the limitation of being a retrospective study solely on outpatients with back pain and did not include patients without symptoms. So, patients of the isthmic type had an advanced degeneration respectively compared to general patients of the isthmic type. Other limitations are that the number of patients involved was relatively scarce and only radiological aspects were considered. Furthermore, as the height of disks was calcu- lated on sagittal images of MRI, it could be different with height of disks on standing radiographs. Also, both patients that had surgery and patients that had conservative treatment were included, therefore, in the future a greater effort needs to be put forth towards finding associations with clinical results and to compare with adjacent segmental instability in post-surgical patients.

\section{Conclusions}

In the two most common forms of spondylolithesis, the isthmic type and the degenerative type, radiologic analysis showed no difference in the extent of translation and instability. Also, it showed more occurrences of the most advanced disc degeneration, fifth degree, $\mathrm{SN}$ and Modic change in the lesion segment of the isthmus type. Lastly, HIZ lesions occurred frequently in the segment superior to the lesion segment of the isthmic type compared to the degenerative type. Judging from these findings, we can consider that stabilization of the lesion segment may occur more commonly in the isthmic type of spondylolisthesis than in the degenerative type.

\section{Conflict of interest}

No potential conflict of interest relevant to this article was reported.

\section{Acknowledgments}

This study was supported by research fund from Chosun University Hospital, 2010.

\section{References}

1. Herbiniaux G. Traife'surdivers accouchements laborieux et sur les polypes de la matrice. Brussels: JL Boubers; 1782 .

2. Wiltse LL, Newman PH, Macnab I. Classification of spondylolisis and spondylolisthesis. Clin Orthop Relat Res 1976;(117):23-9.

3. Wiltse LL, Rothman SL. Spondylolisthesis: classification, diagnosis and natural history. Semin Spine Surg 1989;1:78-94.

4. Meyerding HW. Spondylolisthesis. J Bone Joint Surg Am 1931;13:39-48.

5. Saraste H, Brostrom LA, Aparisi T. Prognostic ra- 
diographic aspects of spondylolisthesis. Acta Radiol Diagn (Stockh) 1984;25:427-32.

6. Pfirrmann CW, Metzdorf A, Zanetti M, Hodler J, Boos N. Magnetic resonance classification of lumbar intervertebral disc degeneration. Spine (Phila Pa 1976) 2001;26:1873-8.

7. Modic MT, Steinberg PM, Ross JS, Masaryk TJ, Carter JR. Degenerative disk disease: assessment of changes in vertebral body marrow with MR imaging. Radiology 1988;166:193-9.

8. Knutsson F. The instability associated with disc degeneration in the lumbar spine. Acta Radiol 1944;25:593-609.

9. Fredrickson BE, Baker D, McHolick WJ, Yuan HA, Lubicky JP. The natural history of spondylolysis and spondylolisthesis. J Bone Joint Surg Am 1984;66:699707.

10. Newman PH, Stone KH. The etiology of spondylolisthesis. J Bone Joint Surg Br 1963;45:39-59.

11. Garfin SR. Spinal fusion: the use of bone screws in the vertebral pedicles. Summation. Spine (Phila Pa 1976) 1994;19:2300S-305S.

12. Valkenburg HA, Haanen HM. The epidemiology of low back pain. In: White AA, Gordon SL, editors. American Academy of Orthopaedic Surgeons Symposium. St. Louis: Mosby; 1982.

13. Kirkaldy-Willis WH, Farfan HF. Instability of the lumbar spine. Clin Orthop Relat Res 1982;(165):11023.

14. Fujiwara A, Lim TH, An HS, et al. The effect of disc degeneration and facet joint osteoarthritis on the segmental flexibility of the lumbar spine. Spine (Phila Pa 1976) 2000;25:3036-44.

15. Kim YT, Bin SI, Kang JS, Nah HY. Treatment of spinal stenosis with spondylolisthesis using transpedicular screw systems. J Korean Orthop Assoc 1992;27:1772-84.

16. Moon MS, Lee KS, Lee WC. Clinical evaluation of degenerative spondylolisthesis. J Korean Orthop Assoc 1984;19:640-8.

17. Rosenberg NJ. Degenerative spondylolisthesis. Predisposing factors. J Bone Joint Surg Am 1975;57:46774.

18. Fujiwara A, Tamai K, Yamato M, et al. The relationship between facet joint osteoarthritis and disc de- generation of the lumbar spine: an MRI study. Eur Spine J 1999;8:396-401.

19. Oegema TR Jr, Bradford DS. The inter-relationship of facet joint osteoarthritis and degenerative disc disease. Br J Rheumatol 1991;30 Suppl 1:16-20.

20. Panjabi MM, Krag MH, Chung TQ. Effects of disc injury on mechanical behavior of the human spine. Spine (Phila Pa 1976) 1984;9:707-13.

21. Weishaupt D, Zanetti M, Hodler J, et al. Painful lumbar disk derangement: relevance of endplate abnormalities at MR imaging. Radiology 2001;218:420-7.

22. Aprill C, Bogduk N. High-intensity zone: a diagnostic sign of painful lumbar disc on magnetic resonance imaging. Br J Radiol 1992;65:361-9.

23. Yu SW, Sether LA, Ho PS, Wagner M, Haughton VM. Tears of the anulus fibrosus: correlation between MR and pathologic findings in cadavers. AJNR Am J Neuroradiol 1988;9:367-70.

24. Ricketson R, Simmons JW, Hauser BO. The prolapsed intervertebral disc. The high-intensity zone with discography correlation. Spine (Phila Pa 1976) 1996;21:2758-62.

25. Adams MA, Freeman BJ, Morrison HP, Nelson IW, Dolan P. Mechanical initiation of intervertebral disc degeneration. Spine (Phila Pa 1976) 2000;25:1625-36.

26. Adams MA, Roughley PJ. What is intervertebral disc degeneration, and what causes it? Spine (Phila Pa 1976) 2006;31:2151-61.

27. Rajasekaran S, Babu JN, Arun R, Armstrong BR, Shetty AP, Murugan S. ISSLS prize winner: A study of diffusion in human lumbar discs: a serial magnetic resonance imaging study documenting the influence of the endplate on diffusion in normal and degenerate discs. Spine (Phila Pa 1976) 2004;29:2654-67.

28. Wu HT, Morrison WB, Schweitzer ME. Edematous Schmorl's nodes on thoracolumbar MR imaging: characteristic patterns and changes over time. Skeletal Radiol 2006;35:212-9.

29. Mok FP, Samartzis D, Karppinen J, Luk KD, Fong DY, Cheung KM. ISSLS prize winner: prevalence, determinants, and association of Schmorl nodes of the lumbar spine with disc degeneration: a populationbased study of 2449 individuals. Spine (Phila Pa 1976) 2010;35:1944-52. 